\title{
Specific Heats of Oxygen at Coexistence*
}

\author{
Robert D. Goodwin** and Lloyd A. Weber** \\ Institute for Basic Standards, National Bureau of Standards, Boulder, Colorado 80302
}

(August 8, 1968)

\begin{abstract}
Specific heats of saturated liquid, along the coexistence path, are useful for computing thermodynamic properties throughout the compressed liquid phase. We report 86 experimental heat capacities of oxygen for the two-phase system, liquid plus vapor, from the triple-point to near the critical-point and corresponding derived values for the liquid phase. These results are represented by a formula which can be integrated for heat absorbed and for entropy. The changes in value of internal energy, enthalpy, and entropy of the saturated liquid are tabulated from the triple-point to the critical-point.

Key Words: Coexistence; experimental; heat capacity; liquid phase; oxygen; saturated liquid; specific heat.
\end{abstract}

\section{List of Symbols}

$A, B, C, r$ constants for eq (5.1).

$A_{i}$

$B_{i}$

$C_{i}$

$D_{i}, E_{i}$

$C_{a}$

$C_{b}$

$\bar{C}$

$\bar{C}_{v}$

$C_{\sigma}$

E

$E_{b}$

$E_{s}$

$H$

$\Delta H_{v}$

$J$

$k$

$N$

$N_{b}$

$N_{c}$

$P$

$Q$

$Q / \Delta T$

$R_{b}$

formula.

for curvature. stant total volume.

internal energy.

volt-box output potential. heater current.

enthalpy.

heat of vaporization.

the joule. tube. coefficients for liquid density formula.

coefficients for rectilinear diameter

coefficients of formula for $C_{b}$.

binomial coefficients (appendix IV).

gross heat capacity adjusted for curvature.

heat capacity of empty calorimeter bomb.

specific heat, two-phase sample, adjusted

specific heat of two-phase sample at con-

specific heat of liquid on coexistence path.

potential across standard resistor for

conversion factor, $0.101325 \mathrm{~J} / \mathrm{cm}^{3}$ atm.

total g moles of fluid in bomb plus capillary

g moles of fluid in the bomb.

$\mathrm{g}$ moles of fluid in the capillary tube.

pressure, $1 \mathrm{~atm}=0.101325 \mathrm{MN} / \mathrm{m}^{2}$.

heat energy; also calorimetric heat input. unadjusted, gross heat capacity.

volt-box input resistance.

\footnotetext{
*This work was carried out at the National Bureau of Standards under the sponsorship of the National Aeronautics and Space Administration Fund Transfer R-06-006-046.

${ }^{* *}$ Cryogenics Division, NBS Boulder Laboratories, Boulder, Colorado 80302

${ }^{1}$ Figures in brackets indicate the literature references at the end of this paper.
}

$R_{l} \quad$ input lead-wire resistance.

$\rho$ density.

$S \quad$ entropy.

$t \quad$ time.

$T \quad$ temperature, Kelvin.

$T_{1}, T_{2} \quad$ initial and final temperatures of a heating interval.

$T_{a} \quad$ average temperature in $\Delta T$.

$T_{c} \quad$ critical-point temperature, $154.77 \mathrm{~K}$. [17]

$T_{t} \quad$ triple-point temperature, $54.3507 \mathrm{~K}$. [17, 18]

$\Delta T \quad$ calorimetric temperature increment, deg.

Celsius.

$\begin{array}{ll}v & \text { molal volume, } 1 / \rho . \\ V_{b} & \text { volume of the calorimeter bomb. }\end{array}$

$V_{l} \quad$ total liquid volume.

\section{Introduction}

This work is part of a program on thermodynamic properties of oxygen. For gaseous states the temperature dependence of these properties may be obtained from spectroscopically derived specific heats at zero density $[1]^{1}$ combined with accurate $P V T$ data. For compressed liquid states, however, additional data are required. Useful among these are specific heats of saturated liquid, $C_{\sigma}(T)$, along the coexistence path. In the present report we give experimental results from near the triple-point to near the criticalpoint. We give an empirical formula for temperature dependence of these results, and the integrals in analytical form which we use for calculating the heat absorbed and the change in value of entropy on this path. We tabulate also the energy and enthalpy. Our results for specific heats in single-phase domains will be reported separately. In a forthcoming publication by one of us (L.A.W.), the present results are used 
with extensive $P V T$ data to construct a complete thermodynamic network for oxygen from the triplepoint to $300 \mathrm{~K}$ at pressures to $300 \mathrm{~atm}$.

\section{Apparatus and Procedures}

\subsection{Apparatus}

The calorimeter is the same as used previously for hydrogen $[2,3]$, except that nearly all of the internal electric wires were replaced (following a relocation of our laboratory). In brief, the spherical sample-holder of type 316 stainless steel is about 2 in in diameter. It is filled via a stainless steel capillary tube 34 in long, $\frac{1}{16}$ in o.d. and 0.006 in i.d., terminating in a shutoff valve on top of the cryostat. A lightweight, cylindrical copper case on the sphere protects the $100 \Omega$ electric heater (wound on the sphere) and serves as the reference temperature for two thermopiles $(100 \mu \mathrm{V} / \mathrm{deg} \mathrm{C})$ controlling a guard ring and the adiabatic shield. For control of each of these shields we use a commercial, electronic "controller," giving automatic reset and rate sensitivity, in addition to our previous equipment. We observe the relative shield temperatures continuously on a recorder.

Temperature of the sample-holder is measured with the same platinum resistance thermometer (NBS 1955 scale) and 6-dial, $\mu \mathrm{V}$ potentiometer. Pressures are measured with the same dead-weight gage (piston diameters recently recalibrated), accurate to about one part in 10,000 . For safety, oil of the gage is separated from the oxygen by nitrogen gas between two null-diaphragms. Filling pressures are corrected for hydrostatic pressure of cold oxygen in the capillary tube.

We obtain the calorimetric heating rate from simultaneous readings of potential and current. $\mathrm{Po}_{0}$ tential wires are connected at the heater in a manner to account for heat developed in the current-carrying wires between shields and calorimeter $[4,5]$. The potential wires lead to a potential-divider (volt-box). We correct for resistance of these potential wires, and for current drawn by the volt-box. The heater current is measured potentiometrically by means of a calibrated, standard resistor and a type $\mathrm{K}-3$ potentiometer.

Time of the heating interval, $\Delta t$, is measured by an electronic counter (quartz piezoelectric oscillator) accurate to better than one part in a million. This counter is switched on and off by the potential across the calorimeter heater. An intervening transmitting circuit, of 22 megohm input impedance, gives the counter a signal independent of the heater potential.

\subsection{Procedures for the Sample}

The commercial high-purity oxygen (maximum nominal impurities $0.01 \%$ ) is taken from a steel cylinder at about $130 \mathrm{~atm}$. At this pressure the oxygen flows through a molecular sieve trap at $76 \mathrm{~K}$, and is admitted slowly to the calorimeter bomb until this pressure also is near $130 \mathrm{~atm}$. The amount of sample is fixed by the selected bomb temperature. A steady state exists after $30 \mathrm{~min}$, when we measure temperature and pressure. We then immediately close the cryostat valve, and begin cooling the bomb into the region of two phases for the contained oxygen.

\subsection{Procedure for a Heat Measurement}

We measure five temperatures, $T(t)$, in several minutes immediately preceding a heat. They are extrapolated linearly to obtain $T_{1}$ at the midtime, $t_{a}$, of the heating interval. For a temperature rise of about $5{ }^{\circ} \mathrm{C}$ in $10 \mathrm{~min}$ we use currents from 0.04 to $0.12 \mathrm{~A}$, dependent on the amount of sample. We average electric power from three pairs of potential and current readings. Shield temperatures as shown on the recorder lag about $0.005{ }^{\circ} \mathrm{C}$ at the start, recovering to within $0.001{ }^{\circ} \mathrm{C}$ of the calorimeter in about a minute. The opposite behavior follows ending of a heat. We then observe temperature drift of the calorimeter on another recorder. In about $20 \mathrm{~min}$ the temperature no longer increases, and may begin a very slow decrease (imperfect adiabatic shielding), when we take another five values for $T(t)$ over about $5 \mathrm{~min}$. They are extrapolated linearly to obtain $T_{2}$ at $t_{a}$.

\section{Calculations and Adjustments}

\subsection{Amount of Sample}

Given the filling conditions $P$ and $T$, we use an equation of state, appendix $\mathrm{V}$, to calculate the onephase density with an uncertainty of about 0.1 percent. By use of our bomb volume (uncertainty $0.1 \%$, appendix I) we then have the amount of sample in the bomb. We sum amounts over the length of the capillary tube by use of estimated temperatures in appendix II, and above equation of state. Sum of amounts in bomb and capillary is $N$, total gram moles in the closed system, with maximum uncertainty of 0.2 percent.

\subsection{The PVT Conditions}

We use the average temperature for a specific heat observation, $T_{a}=\left(T_{1}+T_{2}\right) / 2$, to calculate pressure $P_{a}$ from the vapor-pressure equation (appendix VI). First and second derivatives of $P(T)$ also are calculated at $T_{a}$. Volume of the bomb, $V_{b}$, and $d V_{b} / d T$ are calculated at $T_{a}, P_{a}$ (appendix I). Amount of sample in the bomb is $N_{b}=N-N_{c}$ where $N_{c}$ is amount in the capillary tube at $T_{a}, P_{a}$. The increased amount in capillary tube, $\delta N_{c}$, is amount at $T_{2}, P_{2}$ less amount at $T_{1}, P_{1}$. Average density of the sample is defined $\rho_{\text {av }} \equiv N_{b} / V_{b}$.

Density of the liquid phase, $\rho_{l} \mathrm{~mole} / \mathrm{cm}^{3}$, and the derivative versus $T$, we obtain from the function (L.A.W.) of table 1. By use of the function for rectilinear diameter (L.A.W.) table 2 , we obtain the vapor density, $\rho_{g}$. Relative volume of liquid in the bomb then is

$$
V_{l} / V_{b}=\left(\rho_{\mathrm{av}}-\rho_{g}\right) /\left(\rho_{l}-\rho_{g}\right),
$$


where $V_{l}$ is absolute (not molal) volume of the liquid phase.

\subsection{Gross, Tare, and Net Heat Capacities}

Corresponding to the temperature rise $\Delta T$, the total heat absorbed is $Q$ joules, calculated from volt-box potential $E_{b}$, potential $E_{s}$ across the standard resistor (both in volts), and time $\Delta t$, seconds,

$$
\begin{gathered}
E_{x} \equiv E_{b} \cdot\left(1+R_{l} / R_{b}\right) / K, \\
I_{b}=E_{x} /\left(R_{b}+R_{l}\right), \\
Q=E_{x} \cdot\left[E_{s} / R_{s}-I_{b}\right] \cdot \Delta t .
\end{gathered}
$$

In (3.4), $R_{s}=1.000004 \Omega, R_{b}=225,000 \Omega$ is volt-box input resistance, $R_{l}=30 \Omega$ is resistance of the potential wires between heater and volt-box, and $K=1 / 2000$ is the volt-box ratio $(0.02 \%$ accuracy). Potentiometer accuracies for $E_{b}$ and $E_{s}$ are 0.01 percent and 0.015 percent. Uncertainty in $Q$ therefore is about 0.05 percent.

TABLE 1. Densities $\rho_{l}$ of saturated liquid oxygen

\begin{tabular}{|c|c|}
\hline 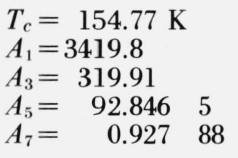 & $\begin{array}{l}\rho_{c}=0.01362 \mathrm{~mol} / \mathrm{cm}^{3} \\
A_{2}=516.52 \\
A_{4}=-275.099 \\
A_{6}=-14.724 \quad 0\end{array}$ \\
\hline
\end{tabular}

$$
\rho_{l}=\rho_{c}+10^{-6} \cdot \sum_{n=1}^{7} A_{n} \cdot x^{n}
$$

where $x \equiv\left(T_{c}-T\right)^{1 / 3}$.

TABLE 2. Rectilinear diameter for oxygen

$$
\left(\rho_{l}+\rho_{g}\right) / 2=\rho_{c}+10^{-3} \cdot \sum_{n=1}^{8} B_{n} \cdot x^{n}
$$

where $x \equiv\left(T_{c}-T\right) / 100$.

\begin{tabular}{l|l}
\hline \hline & \\
$T_{c}=154.77 \mathrm{~K}$ & $\rho_{c}=0.01362 \mathrm{~mol} / \mathrm{cm}^{3}$ \\
$B_{1}=5.892$ & $B_{2}=3.256$ \\
$B_{3}=-14.031$ & $B_{4}=43.814$ \\
$B_{5}=-75.269$ & $B_{6}=71.788$ \\
$B_{7}=-36.405$ & $B_{8}=7.727$
\end{tabular}

As discussed below, the value of $\Delta T$ depends on the temperature scale, on extrapolations in time, and randomly on use of the potentiometer dials. We take the nominal uncertainty in $\Delta T$ to be $(0.1 \%) \cdot(5 / \Delta T)$. Uncertainty in the observed gross heat capacity, $Q / \Delta T$, therefore is roughly 0.15 percent.

An uncertainty in $\Delta T$ of roughly 0.05 percent is probable, based on uncertainties in slope of the temperature scale relative to the thermodynamic scale [7]. The statistical uncertainty in temperature increment, $\Delta T=T_{2}-T_{1}$, obtained from least squares fitting of the $T(t)$ data, usually is near 0.01 percent, with rare values of 0.03 percent. Possible uncertainties arising from accuracy of the potentiometer for $\Delta T=5.0{ }^{\circ} \mathrm{C}$, are given by the last column of table 3 . Values this large arise only by chance if the uppermost dial of the potentiometer is changed in obtaining $\Delta T$. Most frequently the uncertainties will be 0.1 to 0.01 of these values. R. E. Barieau kindly pointed out that this uncertainty could be diminished by an autocalibration of the potentiometer.

TABLE 3. Uncertainties of temperatures derived from the potentiometer accuracy, $\mathrm{k}=0.01$ percent, and characteristics of the platinum resistance thermometer

\begin{tabular}{c|c|c|c|c}
\hline \hline \multicolumn{5}{c}{$\delta T=k \cdot R \cdot d T / d R$} \\
\hline \multirow{2}{*}{$T, \mathrm{~K}$} & $R, \Omega$ & $d R / d T$ & $\delta T$ & $(\delta T / 5.0), \%$ \\
& & & & \\
50 & 1.923 & 0.095 & 0.002 & 0.04 \\
100 & 7.305 & .110 & .007 & .13 \\
150 & 12.72 & .107 & .012 & .24 \\
200 & 17.99 & .104 & .017 & .35 \\
300 & 28.23 & .101 & .028 & .56 \\
\hline
\end{tabular}

We measured 68 tare heat capacities $C_{b}$ of the empty calorimeter bomb from 55 to $300 \mathrm{~K}$, in $\mathrm{J} / \mathrm{K}$. They were adjusted for curvature by application of a cubic expression in three separate ranges of $T$. After investigating various ways to utilize Debye functions, we represent these data more simply by

$$
\log _{e}\left(C_{b} / 50\right)=\sum_{i=1}^{8} C_{i} \cdot(100 / T)^{i-1},
$$

with an rms relative deviation of 0.11 percent. Table 4 gives values for coefficients, $C_{i}$. For this report the deviation applies to values of $C_{b}$ from 20 to $67 \mathrm{~J} / \mathrm{K}$ in the range $55 \leqslant T<155 \mathrm{~K}$. For any quantity of sample, therefore, absolute uncertainty in the quantity $\left[Q / \Delta T-C_{b}\right]$ will range from 0.02 to $0.07 \mathrm{~J} / \mathrm{K}$ due to uncertainties in $C_{b}$ alone over the above range of $T$.

TABLE 4. Constants for $\mathrm{C}_{\mathrm{b}}$ of the empty calorimeter, eq

$(3.5)$

\begin{tabular}{ll|ll}
\hline \hline & & & \\
$C_{1}=0.9625$ & 75 & $C_{2}=-2.6479$ & 48 \\
$C_{3}=6.6945$ & 41 & $C_{4}=-11.5636$ & 585 \\
$C_{5}=10.9871$ & 61 & $C_{6}=-6.0136$ & 545 \\
$C_{7}=1.7776$ & 616 & $C_{8}=-0.2195$ & 491 \\
\hline
\end{tabular}

\subsection{Curvature Adjustment}

If the specific heat is not a linear function of $T$, a curvature adjustment may be necessary to find its value at the average temperature $T_{a}[5]$. We have applied this adjustment to the gross heat capacities, assuming that their true behavior is represented by

$C_{a}=C_{1}+C_{2} \cdot T_{a}+C_{3} \cdot T_{a}^{2}+C_{4} \cdot T_{a}^{3}+C_{5} / \sqrt{T_{c}-T_{a}}$, 
where $T_{c}$ is the critical temperature. (The coefficients $C_{1}, C_{2}$ etc. are not related to those of table 4 .) The experimental gross heat capacity at average temperature $T_{a}$ then is

$$
\begin{aligned}
C_{a}= & Q / \Delta T \\
& -\left\{C_{3} / 12+C_{4} \cdot T_{a} / 4+\left(C_{5} / 32\right) /\left(T_{c}-T_{a}\right)^{5 / 2}\right\} \cdot \overline{\Delta T}^{2} .
\end{aligned}
$$

This formula is obtained by integrating (3.6), i.e., $C_{a} \equiv \delta Q / d T$, between the limits $\left(T_{a}-\Delta T / 2\right)$ and $\left(T_{a}+\Delta T / 2\right)$. Collection of terms then yields two parts for $Q, C_{a}\left(T_{a}\right)$ the desired heat capacity at $T_{a}$, and a correction term which is a function of $T_{a}$ and of $\Delta T$. Coefficients in (3.6) are found by least squares, using $C_{a}=Q / \Delta T$. The difference between $C_{a}$ and $Q / \Delta T$ is found to be small compared with various uncertainties and therefore could be neglected in the present work. For brevity, we define results of the curvature adjustment on specific heat of the two-phase sample as follows (prior to adjustment for calorimeter volume expansion),

$$
\bar{C}=\left(C_{a}-C_{b}\right) / N_{b},
$$

with uncertainty of roughly 0.5 percent when $C_{a} \approx 2 \cdot C_{b}$.

\subsection{Expansion and Capillary Adjustments}

The bomb expands during the calorimetric interval $\Delta T$. An adjustment to find specific heat at constant volume has been described $[8,9]$. The capillary tube absorbs an amount of vapor, $\delta N_{c}$, which must boil away from liquid in the bomb. Combining these adjustments we obtain $C_{v}$ for the two-phase sample,

$\bar{C}_{v}=\bar{C}-\left\{T_{a}(d P / d T)\left(d V_{b} / d T\right)+\delta N_{c} \cdot \Delta H_{v} / \Delta T\right\} / N_{b}$.

For this expression, we describe the heat of vaporization in joules per mole by use of $x \equiv\left(1-T_{a} / T_{c}\right)$,

$$
\Delta H_{v}=x^{0.4} \cdot(1.05-0.2 \cdot x) \cdot 10^{4}
$$

For computing overall uncertainty (see below), we take the following rough estimates,

$$
\begin{array}{cc}
(d P / d T), 0.5 \%, & (d V / d T), 2.0 \% \\
\delta N_{c}, 100.0 \%, & \Delta H_{v}, 2.0 \% .
\end{array}
$$

A large uncertainty is assumed for $\delta N_{c}$ because temperature along the capillary tube is poorly defined. A portion of the total capillary volume of $0.015 \mathrm{~cm}^{3}$ is outside the cryostat, at room temperature.

\subsection{Calculation for $\mathbf{C}_{\sigma}$}

At sufficiently low temperatures one may observe directly $C_{\sigma}$, the heat capacity of the condensed phase at its own (negligible) vapor pressure. Otherwise $C_{\sigma}$ is related to $\vec{C}_{v}$ of the two-phase system via PVT properties $[8,10]$

$C_{\sigma}=\bar{C}_{v}+(T / \rho)$

$\cdot\left\{(-d \rho / d T)(d P / d T) / \rho-\left(\left(V_{b} \cdot \rho / N_{b}\right)-1\right) \cdot d^{2} P / d T^{2}\right\}$,

where $\rho$ referes to the liquid phase. At the critical point $(-d \rho / d T)=\infty$. If the bomb is completely filled with liquid, $V_{b} \cdot \rho / N_{b}=1$. For computing overall uncertainty (see below), we take the following rough estimates

$$
\begin{array}{ll}
\rho, 0.1 \% & V_{b} / N_{b}, 0.2 \% \\
d \rho / d T, 1.0 \% & d^{2} P / d T^{2}, 5.0 \%
\end{array}
$$

\section{Experimental Results}

Table 5 gives loading conditions for the sample in five experimental runs. Temperature and pressure, obtained by computation from laboratory observations, in effect are direct measurements. Volume of the bomb is computed, appendix I. Density is obtained by iterating the equation of state [6]. Total g moles, $N$, includes a few ten-thousandths in theicapillary tube.

Table 6 gives the experimental conditions, the specific heats, and the uncertainties (errors) for each measurement (point) of the five experimental runs. Under ID is given the run number, followed by two digits for the point in that run. Temperature is the average, $T_{a}$. Pressure, bomb volume, and density are calculated, section 3 . This density is the defined average, $\rho_{\text {av }}=N_{b} / V_{b}$. The sixth column gives $\Delta T$, followed by $Q / \Delta T$, its value adjusted for curvature, and the tare heat capacity from (3.5). Next is $\bar{C}_{v}$ for the two-phase system, obtained via the adjustment of (3.9), and then $C_{\sigma}$ calculated via (3.11), both in $\mathrm{J} /(\mathrm{mol} \mathrm{K})$. The last two columns give uncertainties for $\bar{C}_{v}$ and for $C_{\sigma}$ in percent, calculated by methods of appendix III.

TABLE 5. Loading conditions for the samples

\begin{tabular}{r|r|r|r|r|r}
\hline \hline \multirow{2}{*}{ Run } & \multicolumn{1}{|l|}{$T, \mathrm{~K}$} & \multicolumn{1}{l|}{$P, \mathrm{~atm}$} & $\boldsymbol{V}, \mathrm{cm}^{3}$ & $\rho, \mathrm{mol} / \mathrm{l}$ & $N, \mathrm{~mol}$ \\
\hline & & & & & \\
2 & 197.279 & 131.432 & 73.133 & 13.128 & 0.9602 \\
3 & 139.255 & 129.352 & 72.950 & 28.679 & 2.0924 \\
5 & 173.070 & 129.980 & 73.054 & 20.223 & 1.4775 \\
20 & 180.928 & 123.245 & 73.072 & 16.833 & 1.2302 \\
32 & 137.181 & 106.345 & 72.934 & 28.633 & 2.0882 \\
\hline
\end{tabular}


TABLE 6. Experimental specific heats, saturated liquid oxygen

\begin{tabular}{|c|c|c|c|c|c|c|c|c|c|c|c|c|}
\hline \multirow[t]{2}{*}{ ID } & \multirow{2}{*}{$\underset{K}{\text { Temp. }}$} & \multirow{2}{*}{$\begin{array}{l}\text { Pres. } \\
\text { atm. }\end{array}$} & \multirow{2}{*}{$\begin{array}{c}V_{b} \\
\mathrm{~cm}^{3}\end{array}$} & \multirow{2}{*}{$\begin{array}{l}\text { Dens. } \\
\mathrm{mol} / \mathrm{l}\end{array}$} & \multirow{2}{*}{$\begin{array}{c}\text { DEL } T \\
K\end{array}$} & \multirow{2}{*}{$\begin{array}{c}D Q / D T \\
\mathrm{~J} / \mathrm{deg}\end{array}$} & \multirow{2}{*}{$\begin{array}{c}D Q D T A \\
\mathrm{~J} / \mathrm{deg}\end{array}$} & \multirow{2}{*}{$\begin{array}{l}\text { Tare } \\
\mathrm{J} / \mathrm{deg}\end{array}$} & $C_{v}$ & CSAT & Errors & Percent \\
\hline & & & & & & & & & M-K & $\mathrm{J} / \mathrm{M}-\mathrm{K}$ & $C_{V}$ & CSAT \\
\hline 201 & 56.948 & 0.003 & 72.669 & 13.214 & 2.754 & 74.096 & 74.096 & 22.910 & 53.307 & 53.243 & 0.580 & 0.587 \\
\hline 202 & 60.157 & .007 & 72.672 & 13.213 & 3.673 & 76.465 & 76.466 & 25.185 & 53.404 & 53.280 & .527 & .541 \\
\hline 203 & 60.357 & .008 & 72.672 & 13.213 & 3.645 & 76.628 & 76.629 & 25.326 & 53.428 & 53.298 & .529 & .543 \\
\hline 204 & 63.936 & .018 & 72.677 & 13.212 & 3.525 & 79.350 & 79.350 & 27.835 & 53.649 & 53.405 & .550 & .577 \\
\hline 205 & 67.925 & .042 & 72.682 & 13.211 & 4.481 & 82.261 & 82.261 & 30.578 & 53.824 & 53.377 & .516 & .566 \\
\hline 206 & 72.313 & .092 & 72.688 & 13.210 & 4.309 & 85.477 & 85.477 & 33.501 & 54.129 & 53.345 & .538 & .626 \\
\hline 207 & 76.537 & .181 & 72.695 & 13.209 & 4.151 & 88.651 & 88.650 & 36.199 & 54.624 & 53.390 & .558 & .697 \\
\hline 208 & 80.914 & .335 & 72.702 & 13.208 & 4.614 & 92.088 & 92.087 & 38.860 & 55.430 & 53.591 & .548 & .756 \\
\hline 247 & 81.735 & .374 & 72.704 & 13.207 & .936 & 92.488 & 92.488 & 39.344 & 55.344 & 53.376 & 1.291 & 1.543 \\
\hline 209 & 85.426 & .590 & 72.710 & 13.206 & 4.427 & 95.857 & 95.856 & 41.459 & 56.648 & 54.041 & .564 & .860 \\
\hline 248 & 83.340 & .458 & 72.707 & 13.207 & 2.276 & 93.826 & 93.826 & 40.276 & 55.767 & 53.532 & .749 & 1.011 \\
\hline 210 & 87.465 & .745 & 72.714 & 13.205 & 4.377 & 97.515 & 97.513 & 42.585 & 57.201 & 54.201 & .570 & .911 \\
\hline 211 & 91.759 & 1.176 & 72.723 & 13.204 & 4.228 & 100.793 & 100.791 & 44.859 & 58.246 & 54.336 & .585 & 1.032 \\
\hline 212 & 95.904 & 1.752 & 72.732 & 13.202 & 4.088 & 104.017 & 104.014 & 46.933 & 59.441 & 54.561 & .598 & 1.160 \\
\hline 213 & 100.224 & 2.557 & 72.742 & 13.200 & 4.580 & 107.561 & 107.558 & 48.972 & 61.006 & 55.036 & .578 & 1.266 \\
\hline 214 & 104.683 & 3.649 & 72.752 & 13.198 & 4.432 & 111.194 & 111.190 & 50.953 & 62.724 & 55.572 & .588 & 1.417 \\
\hline 215 & 109.022 & 5.011 & 72.763 & 13.196 & 4.280 & 114.936 & 114.931 & 52.764 & 64.730 & 56.397 & .597 & 1.567 \\
\hline 216 & 113.688 & 6.856 & 72.776 & 13.194 & 5.087 & 118.950 & 118.942 & 54.592 & 66.999 & 57.402 & .564 & 1.682 \\
\hline 217 & 118.664 & 9.323 & 72.790 & 13.192 & 4.899 & 123.492 & 123.484 & 56.412 & 69.827 & 58.951 & .569 & 1.847 \\
\hline 218 & 123.185 & 12.070 & 72.803 & 13.189 & 3.846 & 127.721 & 127.715 & 57.958 & 72.617 & 60.725 & .619 & 2.043 \\
\hline 219 & 127.626 & 15.294 & 72.817 & 13.187 & 5.079 & 132.040 & 132.026 & 59.383 & 75.615 & 62.979 & .558 & 2.091 \\
\hline 220 & 132.584 & 19.576 & 72.834 & 13.183 & 4.881 & 137.197 & 137.178 & 60.872 & 79.419 & 66.468 & .561 & 2.204 \\
\hline 221 & 137.495 & 24.605 & 72.851 & 13.180 & 5.115 & 142.744 & 142.710 & 62.249 & 83.733 & 71.438 & .547 & 2.265 \\
\hline 222 & 143.712 & 32.240 & 72.875 & 13.176 & 5.273 & 151.443 & 151.349 & 63.863 & 91.026 & 82.803 & .532 & 2.274 \\
\hline 223 & 148.798 & 39.696 & 72.896 & 13.172 & 4.954 & 161.666 & 161.307 & 65.087 & 100.099 & 104.910 & .529 & 2.174 \\
\hline 224 & 152.389 & 45.729 & 72.912 & 13.169 & 2.288 & 174.037 & 173.290 & 65.903 & 111.709 & 159.122 & .702 & 2.035 \\
\hline 301 & 57.563 & .004 & 72.669 & 28.793 & 2.150 & 134.441 & 134.440 & 23.348 & 53.094 & 53.080 & .563 & .565 \\
\hline 302 & 60.020 & .007 & 72.672 & 28.792 & 2.769 & 136.329 & 136.328 & 25.089 & 53.164 & 53.142 & .505 & .508 \\
\hline 303 & 63.138 & .015 & 72.676 & 28.790 & 3.473 & 138.737 & 138.735 & 27.279 & 53.268 & 53.229 & .466 & .471 \\
\hline 304 & 67.026 & .035 & 72.681 & 28.788 & 4.309 & 141.548 & 141.546 & 29.966 & 53.327 & 53.259 & .438 & .446 \\
\hline 305 & 71.253 & .077 & 72.687 & 28.786 & 4.146 & 144.316 & 144.314 & 32.805 & 53.293 & 53.179 & .450 & .466 \\
\hline 306 & 71.610 & .082 & 72.687 & 28.786 & 4.145 & 144.308 & 144.307 & 33.041 & 53.177 & 53.059 & .451 & .467 \\
\hline 307 & 76.180 & .171 & 72.694 & 28.783 & 5.008 & 147.560 & 147.559 & 35.975 & 53.329 & 53.146 & .431 & .456 \\
\hline 308 & 81.112 & .344 & 72.703 & 28.780 & 4.874 & 151.277 & 151.277 & 38.978 & 53.671 & 53.407 & .441 & .480 \\
\hline 309 & 85.910 & .624 & 72.711 & 28.776 & 4.757 & 155.073 & 155.074 & 41.729 & 54.170 & 53.827 & .450 & .504 \\
\hline 310 & 91.013 & 1.090 & 72.721 & 28.772 & 5.466 & 158.927 & 158.929 & 44.473 & 54.700 & 54.289 & .436 & .508 \\
\hline 311 & 96.412 & 1.835 & 72.733 & 28.768 & 5.353 & 162.482 & 162.484 & 47.179 & 55.105 & 54.663 & .444 & .535 \\
\hline 312 & 101.694 & 2.885 & 72.745 & 28.763 & 5.231 & 166.132 & 166.133 & 49.639 & 55.672 & 55.272 & .452 & .560 \\
\hline 313 & 106.854 & 4.290 & 72.758 & 28.758 & 5.115 & 169.825 & 169.823 & 51.873 & 56.367 & 56.113 & .459 & .582 \\
\hline 314 & 111.605 & 5.980 & 72.770 & 28.753 & 5.817 & 173.190 & 173.184 & 53.791 & 57.055 & 57.073 & .445 & .577 \\
\hline 315 & 117.372 & 8.629 & 72.786 & 28.747 & 5.738 & 177.526 & 177.510 & 55.952 & 58.087 & 58.690 & .449 & .587 \\
\hline 316 & 123.021 & 11.961 & 72.803 & 28.740 & 5.585 & 182.011 & 181.979 & 57.904 & 59.286 & 60.874 & .455 & .588 \\
\hline 501 & 5 & .005 & 72.6 & 20.332 & 7.229 & & 102.718 & 24.024 & & & .386 & .391 \\
\hline 502 & 65.588 & .026 & 72.679 & 20.330 & 6.924 & 107.935 & 107.935 & 28.979 & 53.437 & 53.289 & .404 & .421 \\
\hline 503 & 72.387 & .093 & 72.688 & 20.327 & 6.700 & 112.688 & 112.687 & 33.549 & 53.561 & 53.199 & .420 & .462 \\
\hline 504 & 78.969 & .257 & 72.699 & 20.324 & 6.483 & 117.578 & 117.577 & 37.695 & 54.064 & 53.369 & .435 & .517 \\
\hline 505 & 85.485 & .594 & 72.711 & 20.321 & 6.569 & 122.788 & 122.786 & 41.492 & 55.019 & 53.877 & .442 & .579 \\
\hline 506 & 91.909 & 1.194 & 72.723 & 20.317 & 6.302 & 127.571 & 127.569 & 44.936 & 55.924 & 54.261 & .455 & .660 \\
\hline 507 & 98.099 & 2.132 & 72.737 & 20.313 & 6.100 & 132.123 & 132.119 & 47.984 & 56.940 & 54.741 & .466 & .745 \\
\hline 508 & 104.668 & 3.645 & 72.752 & 20.309 & 5.819 & 137.342 & 137.338 & 50.946 & 58.465 & 55.727 & .477 & .840 \\
\hline 509 & 110.671 & 5.615 & 72.767 & 20.305 & 6.216 & 141.950 & 141.943 & 53.424 & 59.901 & 56.770 & .472 & .912 \\
\hline 510 & 116.768 & 8.318 & 72.784 & 20.300 & 6.012 & 147.003 & 146.994 & 55.734 & 61.753 & 58.421 & .479 & .992 \\
\hline 511 & 122.642 & 11.713 & 72.801 & 20.295 & 5.771 & 151.952 & 151.941 & 57.777 & 63.712 & 60.528 & .486 & 1.060 \\
\hline 512 & 128.503 & 15.996 & 72.820 & 20.290 & 6.071 & 157.421 & 157.401 & 59.654 & 66.131 & 63.681 & .479 & 1.096 \\
\hline 513 & 134.442 & 21.382 & 72.840 & 20.284 & 5.848 & 163.348 & 163.314 & 61.404 & 68.939 & 68.327 & .483 & 1.122 \\
\hline 514 & 139.909 & 27.392 & 72.860 & 20.278 & 5.129 & 169.472 & 169.416 & 62.892 & 72.051 & 75.118 & .500 & 1.133 \\
\hline 515 & 144.531 & 33.362 & 72.878 & 20.273 & 4.163 & 175.847 & 175.757 & 64.066 & 75.536 & 85.273 & .532 & 1.133 \\
\hline 2001 & 59.127 & .006 & 72.671 & 16.928 & 4.948 & 90.069 & 90.069 & 24.458 & 53.334 & 53.266 & .445 & .452 \\
\hline 2002 & 63.957 & .018 & 72.677 & 16.927 & 4.748 & 93.761 & 93.761 & 27.849 & 53.578 & 53.414 & .463 & .482 \\
\hline 2003 & 68.641 & .048 & 72.683 & 16.926 & 4.631 & 97.212 & 97.212 & 31.062 & 53.771 & 53.444 & .479 & .516 \\
\hline 2004 & 73.193 & .107 & 72.689 & 16.924 & 4.486 & 100.365 & 100.364 & 34.073 & 53.886 & 53.315 & .496 & .561 \\
\hline 2005 & 77.601 & .212 & 72.697 & 16.922 & 4.348 & 103.626 & 103.625 & 36.858 & 54.273 & 53.377 & .512 & .615 \\
\hline 2006 & 84.131 & .505 & 72.708 & 16.920 & 5.535 & 108.880 & 108.878 & 40.729 & 55.396 & 53.858 & .485 & .662 \\
\hline 2007 & 89.547 & .935 & 72.718 & 16.917 & 5.322 & 113.143 & 113.141 & 43.704 & 56.442 & 54.244 & .499 & .755 \\
\hline 2008 & 95.108 & 1.628 & 72.730 & 16.915 & 5.824 & 117.347 & 117.344 & 46.543 & 57.549 & 54.584 & .492 & .842 \\
\hline
\end{tabular}


TABLE 6. Experimental specific heats, saturated liquid oxygen-Continued

\begin{tabular}{|c|c|c|c|c|c|c|c|c|c|c|c|c|}
\hline \multirow[t]{2}{*}{ ID } & \multirow{2}{*}{$\begin{array}{l}\text { Temp. } \\
\qquad K\end{array}$} & \multirow{2}{*}{$\begin{array}{l}\text { Pres. } \\
\text { atm. }\end{array}$} & \multirow{2}{*}{$\begin{array}{c}V_{b} \\
\mathrm{~cm}^{3}\end{array}$} & \multirow{2}{*}{$\begin{array}{l}\text { Dens. } \\
\mathrm{mol} / \mathrm{l}\end{array}$} & \multirow{2}{*}{$\begin{array}{c}\text { DEL } T \\
\quad K\end{array}$} & \multirow{2}{*}{$\begin{array}{c}D Q / D T \\
\mathrm{~J} / \mathrm{deg}\end{array}$} & \multirow{2}{*}{$\begin{array}{c}D Q D T A \\
\text { J/deg }\end{array}$} & \multirow{2}{*}{$\begin{array}{c}\text { Tare } \\
\mathrm{J} / \mathrm{deg}\end{array}$} & \multirow{2}{*}{$\begin{array}{c}C_{v} \\
\mathrm{~J} / \mathrm{M}-\mathrm{K}\end{array}$} & \multirow{2}{*}{$\begin{array}{l}\text { CSAT } \\
\mathrm{J} / \mathrm{M}-\mathrm{K}\end{array}$} & \multirow{2}{*}{$\frac{\text { Errors }}{C_{v}}$} & \multirow{2}{*}{$\frac{\text { Percent }}{\text { CSAT }}$} \\
\hline & & & & & & & & & & & & \\
\hline 2009 & 101.059 & 2.740 & 72.743 & 16.911 & 6.105 & 122.031 & 122.026 & 49.352 & 59.070 & 55.226 & .492 & .952 \\
\hline 2010 & 107.253 & 4.417 & 72.759 & 16.908 & 6.364 & 127.065 & 127.058 & 52.039 & 60.973 & 56.211 & .490 & 1.072 \\
\hline 2011 & 113.476 & 6.763 & 72.775 & 16.904 & 6.116 & 132.161 & 132.153 & 54.512 & 63.100 & 57.499 & .498 & 1.206 \\
\hline 2012 & 119.674 & 9.893 & 72.793 & 16.900 & 6.315 & 137.612 & 137.599 & 56.766 & 65.689 & 59.463 & .495 & 1.318 \\
\hline 2013 & 125.847 & 13.936 & 72.811 & 16.895 & 6.066 & 143.326 & 143.309 & 58.823 & 68.650 & 62.227 & .500 & 1.424 \\
\hline 2014 & 133.607 & 20.556 & 72.837 & 16.889 & 5.756 & 151.059 & 151.028 & 61.166 & 73.007 & 67.580 & .505 & 1.518 \\
\hline 2015 & 139.222 & 26.577 & 72.858 & 16.884 & 5.513 & 157.562 & 157.504 & 62.711 & 77.003 & 74.171 & .507 & 1.546 \\
\hline 2016 & 144.586 & 33.439 & 72.879 & 16.880 & 5.260 & 165.194 & 165.048 & 64.080 & 82.007 & 85.561 & .508 & 1.531 \\
\hline 2017 & 149.672 & 41.101 & 72.900 & 16.874 & 4.960 & 176.417 & 175.696 & 65.289 & 89.660 & 112.651 & .506 & 1.442 \\
\hline 3201 & 56.440 & .003 & 72.668 & 28.736 & 3.670 & 133.850 & 133.848 & 22.547 & 53.301 & 53.290 & .444 & .446 \\
\hline 3202 & 60.313 & .008 & 72.672 & 28.734 & 4.084 & 136.722 & 136.721 & 25.296 & 53.360 & 53.336 & .434 & .437 \\
\hline 3203 & 64.924 & .023 & 72.678 & 28.732 & 4.364 & 140.173 & 140.172 & 28.521 & 53.468 & 53.417 & .432 & 439 \\
\hline 3204 & 69.487 & .056 & 72.684 & 28.730 & 4.772 & 143.206 & 143.205 & 31.631 & 53.431 & 53.337 & .427 & .440 \\
\hline 3205 & 74.304 & .128 & 72.691 & 28.727 & 4.876 & 146.489 & 146.489 & 34.788 & 53.492 & 53.336 & .431 & .453 \\
\hline 3206 & 79.250 & .268 & 72.699 & 28.724 & 5.032 & 150.113 & 150.114 & 37.865 & 53.754 & 53.519 & . .434 & .468 \\
\hline 3207 & 84.378 & .520 & 72.708 & 28.720 & 5.254 & 154.102 & 154.103 & 40.869 & 54.226 & 53.904 & .434 & .483 \\
\hline 3208 & 89.559 & .937 & 72.718 & 28.716 & 5.122 & 157.864 & 157.865 & 43.710 & 54.666 & 54.266 & .443 & .510 \\
\hline 3209 & 94.825 & 1.585 & 72.729 & 28.712 & 5.428 & 161.408 & 161.409 & 46.404 & 55.072 & 54.626 & .441 & .527 \\
\hline 3210 & 100.347 & 2.583 & 72.742 & 28.707 & 5.635 & 165.171 & 165.171 & 49.029 & 55.616 & 55.187 & .441 & .546 \\
\hline 3211 & 105.901 & 3.999 & 72.755 & 28.701 & 5.493 & 169.126 & 169.124 & 51.472 & 56.337 & 56.034 & .448 & .570 \\
\hline 3212 & 111.610 & 5.982 & 72.770 & 28.696 & 5.321 & 173.243 & 173.237 & 53.793 & 57.193 & 57.196 & .456 & .590 \\
\hline 3213 & 117.010 & 8.441 & 72.785 & 28.690 & 5.502 & 177.342 & 177.328 & 55.821 & 58.178 & 58.716 & .454 & .593 \\
\hline 3214 & 122.534 & 11.642 & 72.801 & 28.683 & 5.572 & 181.691 & 181.663 & 57.741 & 59.331 & 60.791 & .455 & .590 \\
\hline
\end{tabular}

$\mathrm{UN}=0.2, \mathrm{UA}=0.15, \mathrm{UB}=0.1, \mathrm{UC}=2.5, \mathrm{UD}=102.0, \mathrm{UE}=1.6, \mathrm{UF}=0.3, \mathrm{UG}=5.0, \mathrm{UH}=0.1$.

\section{Formulation and Comparisons With Earlier Data}

An analytical description of $C_{\sigma}(T)$ is required for computations. Using the reduced argument, $x \equiv\left(T_{c}-T\right) /\left(T_{c}-T_{t}\right)$ where $T_{c}$ and $T_{t}$ are criticalpoint and triple-point temperatures, we find that a plot of $C_{\sigma} \cdot x^{1 / 2}$ versus $T$ is a straight line, except for a small deviation as $T \rightarrow T_{c}$, figure 1 . In the following

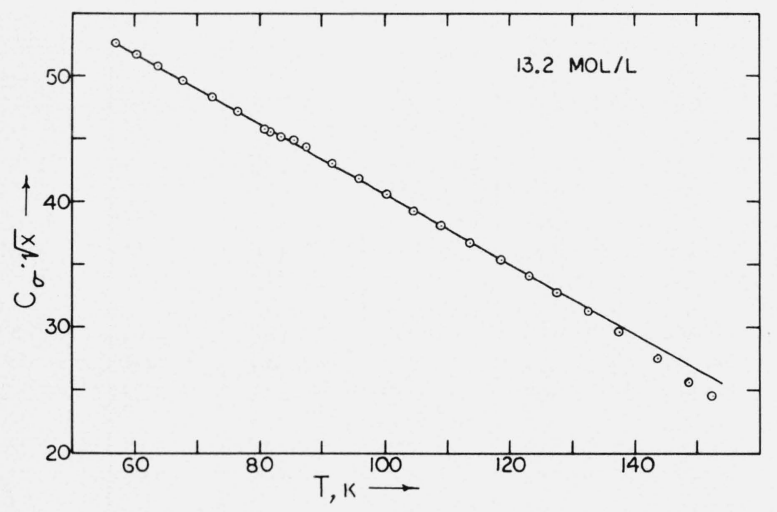

FigURE 1. Data of run No. 2 in coordinates of eq (5.1). form, this deviation is described by a binomial, which can be expanded for term-by-term integration,

$$
C_{\sigma}=\left[A+B \cdot x+C \cdot(1-r \cdot x)^{n}\right] / x^{1 / 2},
$$

with $n=12$. We define $r \equiv\left(T_{c}-T_{t}\right) / T_{c}$, such that $(1-r \cdot x) \equiv T / T_{c}$.

This formula is similar to results obtained for a van der Waals fluid [16]. The weighted least squares determination of coefficients yields

$$
A=25.60277, \quad B=27.71001, \quad C=-2.48274 \text {. }
$$

In table 7 the column PCNT is percent deviation of data, CSAT, from the calculated value, CALC. Weights, $W T$, are inversely proportional to the square of absolute errors obtained from the last column of table 6 . In the last column of table $7, D_{2} \equiv$ (CSATCALC) ${ }^{2}$ and column entries are $D 2 \cdot W T$. For $n=86$ data, the weighted, mean-square relative deviation is

$$
\left\{\sum^{n}\left(\Delta C_{\sigma} / C_{\sigma}\right)^{2} \cdot W T / \sum^{n} W T\right\}^{1 / 2} \cdot 100=0.19 \%
$$

The correspondingly weighted absolute mean-square deviation is $0.11 \mathrm{~J} / \mathrm{mol} \mathrm{K}$. 
TABLE 7. Comparison of experimental specific heats with values calculated from eq (5.1)

\begin{tabular}{|c|c|c|c|c|c|c|c|c|c|c|c|c|c|}
\hline ID & Temp. & CSAT & CALC & PCNT & Weight & $D 2 * W T$ & ID & Temp. & CSAT & CALC & PCNT & Weight & $D 2^{*} W T$ \\
\hline 3201 & 56.440 & 53.290 & 53.294 & -0.007 & 1.5956 & 0.002 & 2008 & 95.108 & 54.584 & 54.565 & .034 & .2872 & .010 \\
\hline 201 & & & & -.088 & .5287 & .115 & 212 & 95.904 & 54.561 & 54.645 & -.154 & .1675 & .119 \\
\hline 301 & 57.563 & 53.080 & 53.286 & -.386 & .5631 & 2.379 & 311 & 96.412 & 54.663 & 54.698 & -.064 & . 5845 & .072 \\
\hline 501 & 58.515 & 53.218 & 53.280 & -.117 & .9487 & .366 & 507 & 98.099 & 54.741 & 54.884 & -.260 & .3490 & .712 \\
\hline 2001 & 59.127 & 53.266 & 53.277 & -.021 & .7744 & .010 & 213 & 100.224 & 55.036 & 55.143 & -.194 & .1418 & .162 \\
\hline 302 & 60.020 & 53.142 & 53.274 & -.248 & .6574 & 1.146 & 3210 & 100.347 & 55.187 & 55.159 & .051 & .9920 & .079 \\
\hline 202 & 60.157 & 53.280 & 53.274 & .012 & .5978 & .002 & 2009 & 101.059 & 55.226 & 55.253 & -.049 & .2298 & .017 \\
\hline 3202 & 60.313 & 53.336 & 53.273 & .118 & 1.6550 & .652 & 312 & 101.694 & 2 & 55.340 & -.123 & .5364 & .247 \\
\hline 203 & 60.357 & 53.298 & 53.273 & .047 & .5929 & .037 & 508 & 104.668 & 55.727 & 55.787 & -.108 & .2790 & .102 \\
\hline 303 & 63.138 & 53.229 & 53.272 & -.081 & .7318 & .136 & 214 & 104.683 & 55.572 & 55.790 & -.390 & .1142 & .542 \\
\hline 204 & 63.936 & 53.405 & 53.274 & .246 & .5404 & .927 & 3211 & 105. & 56.034 & 55.994 & .071 & .8828 & .140 \\
\hline 2002 & 6 & 53.414 & 53.274 & .263 & .7054 & 1.380 & 313 & $10 t$ & 56. & 56. & -.090 & .4952 & .125 \\
\hline 3203 & 64.924 & 53.417 & 53.278 & .261 & 1.6381 & 3.168 & 2010 & 107.253 & 56.211 & 56. & -.045 & .1823 & .012 \\
\hline 502 & 65.588 & 53.289 & 53.281 & .014 & .8563 & .005 & 215 & 109.022 & 56.397 & 56.581 & -.324 & .0930 & .313 \\
\hline 304 & 67.026 & 53.259 & 53.292 & -.061 & .7887 & .085 & 509 & 110.671 & 56.770 & 56.931 & -.283 & 2360 & .612 \\
\hline 205 & 67.925 & 53.377 & 53 & .144 & .556 & .32 & 314 & 111 & 73 & 57.143 & -.123 & . 4881 & .242 \\
\hline 2003 & 64 & 444 & 53 & .255 & .6369 & 1.181 & 3212 & 111 & & & 990 & .7901 & .209 \\
\hline 3204 & 69.487 & 7 & 53 & .035 & 1.6381 & .05 & 2011 & & 5 & 2 & -.178 & 1430 & .151 \\
\hline 305 & 71.253 & 53.179 & 53.345 & -.310 & .7449 & 2.043 & 216 & 113.688 & 57.402 & 57.656 & -.441 & .0793 & .513 \\
\hline 206 & 72.313 & 53.345 & 53.364 & -.035 & .4783 & .016 & 510 & 116.768 & 58.421 & 58.528 & -.183 & 1950 & .224 \\
\hline 503 & 72.387 & 53.19 & 53.3 & -.311 & .7556 & 2.073 & 3213 & 11 & 6 & 58.603 & 3 & 15 & 946 \\
\hline 200 & & & & -.124 & .5645 & .2 & 315 & & & & -.046 & .4557 & .033 \\
\hline 3205 & 74 & & & -.131 & 1.5432 & .7 & 217 & & & & -.324 & .0637 & .234 \\
\hline 207 & 76.537 & 53.390 & 53.464 & -.139 & .4036 & .223 & 2012 & 119.674 & 59.463 & 59.498 & -.058 & 11152 & .014 \\
\hline 2005 & 77.601 & 53.377 & 53.497 & -.224 & .4906 & .702 & 3214 & 122.534 & 60.791 & 60.622 & .278 & .6991 & 1.988 \\
\hline 50 & 78 & 5 & 53 & -.3 & .6 & 1. & 511 & $1 \%$ & 8 & 60 & -.232 & .1 & .324 \\
\hline 3206 & & 5 & 53 & -.0 & 1.4377 & .18 & 316 & 123 & & 60. & .067 & .4296 & .070 \\
\hline 208 & & & & & & & 218 & & & & -.297 & .0501 & .164 \\
\hline 308 & & & & -.4 & .7097 & 3.37 & 2013 & & & & .058 & .0926 & .012 \\
\hline 247 & 81.735 & 53.376 & 53.652 & -.514 & .1055 & .802 & 219 & 127.626 & 62.979 & 63.179 & -.317 & .0448 & .180 \\
\hline 248 & 83.3 & 53.5 & 53.7 & - & .21 & .82 & 51 & 128. & 63. & 63. & - . & .1474 & .013 \\
\hline 2006 & & & 53.7 & .173 & .43 & .3 & 220 & & & & & & .149 \\
\hline 320 & & & & .2 & 1.3 & 2.11 & 2014 & & & & .022 & .0710 & .002 \\
\hline 209 & & & & .384 & & 1.20 & 513 & & & & -.039 & .1199 & .008 \\
\hline 505 & 85.485 & 53.877 & 53.838 & .073 & .5302 & .082 & 221 & 137.495 & 71.438 & 71.775 & -.470 & .0305 & .347 \\
\hline 309 & 85.91 & 53.8 & 53.8 & -.06 & .65 & .0 & 201 & 139 & 74 & 74. & -.0 & .0 & .005 \\
\hline 210 & & & & .4 & .25 & 1.53 & 51 & & 75. & &.- & .0995 & .300 \\
\hline 2007 & 89.54 & 54.24 & 54.0 & .273 & .3468 & .75 & 22 & & 82. & 83. & -.567 & .0229 & .510 \\
\hline 3208 & & & & .312 & 1.1755 & 3.3 & 515 & & & & -.392 & .0792 & .892 \\
\hline 310 & 91.013 & 54.289 & 54.206 & .153 & .6383 & .441 & 2016 & 144.586 & 85.561 & 85.776 & -.251 & .0453 & .209 \\
\hline 21 & 9 & 54 & 54 & 1 & .20 & .1 & 223 & 148 & 104 & 105 & -.461 & .0159 & .374 \\
\hline 506 & 91.90 & 54.2 & 54.2 & -.031 & .4291 & .012 & 2017 & & & & .132 & .0303 & .067 \\
\hline 3209 & 94.825 & 54.626 & 54.538 & .162 & 1.0881 & .845 & 224 & 152.389 & 159.122 & 157.151 & 1.254 & .0081 & 3.136 \\
\hline
\end{tabular}

Comparison with earlier data, which exist at temperatures up to the boiling-point, becomes possible by use of $(5.1)$ for interpolation. Table 8 gives such data for saturated liquid oxygen, in $\mathrm{J} / \mathrm{mol} \mathrm{K}[11,12$, $4,13]$, and compares them with our calculated results. The mean deviation of a few tenths of 1 percent from the data of Giauque and Johnston is within our accuracy.

Values for $d^{2} P / d T^{2}$ at saturation can be derived from two-phase heat capacities at two or more average densities [10]. Such data may be an aid to formulating an accurate vapor pressure equation, and R. E. Barieau brought this to our attention [14] almost simultaneously with the report of Yang and Yang [15]. For coexisting phases the relation is

$$
\bar{C}_{v} / T=-d^{2} G / d T^{2}+\left(d^{2} P / d T^{2}\right) \cdot v
$$

where $G$ is the Gibbs free energy per mole and $v$ is the average molal volume. We see that $d^{2} P / d T^{2}$ is obtained on isotherms as the slope of plots of $\bar{C}_{v} / T$ versus $v$. We now use this method only to show consistency between our experimental specific heats and available vapor pressure equations of high accuracy. We have compared two vapor pressure equations $[6,7]$ with each other, finding excellent agreement 
in the second derivatives from the triple point to the boiling point.

Column 10 of table 6 gives the raw data, $\bar{C}_{v}$, for five runs at four significantly different average densities.

TABLE 8. Comparison of calculated specific heats, J/mol K, with Euken, 1; Clusius, 2; Giauque, 3; Itterbeek, 4.

\begin{tabular}{|c|c|c|c|c|}
\hline ID & $T, K$ & Refer. & Calcd. & Prent. \\
\hline $\begin{array}{l}1 \\
1 \\
1 \\
1 \\
1\end{array}$ & $\begin{array}{l}57.40 \\
60.50 \\
65.10 \\
67.70 \\
69.50\end{array}$ & $\begin{array}{l}53.60 \\
53.56 \\
53.39 \\
53.22 \\
53.22\end{array}$ & $\begin{array}{l}53.29 \\
53.27 \\
53.28 \\
53.30 \\
53.32\end{array}$ & $\begin{array}{r}0.59 \\
.54 \\
.21 \\
-.15 \\
-.18\end{array}$ \\
\hline $\begin{array}{l}1 \\
1 \\
2 \\
2 \\
2\end{array}$ & $\begin{array}{l}71.30 \\
73.00 \\
56.60 \\
58.00 \\
59.70\end{array}$ & $\begin{array}{l}53.47 \\
52.80 \\
52.80 \\
52.59 \\
52.13\end{array}$ & $\begin{array}{l}53.35 \\
53.38 \\
53.29 \\
53.28 \\
53.28\end{array}$ & $\begin{array}{r}.23 \\
-1.08 \\
-.92 \\
-1.30 \\
-2.15\end{array}$ \\
\hline $\begin{array}{l}2 \\
2 \\
2 \\
2 \\
2\end{array}$ & $\begin{array}{l}62.50 \\
65.10 \\
65.50 \\
67.40 \\
67.60\end{array}$ & $\begin{array}{l}53.72 \\
54.14 \\
52.47 \\
52.26 \\
53.68\end{array}$ & $\begin{array}{l}53.27 \\
53.28 \\
53.28 \\
53.30 \\
53.30\end{array}$ & $\begin{array}{r}.84 \\
1.62 \\
-1.52 \\
-1.94 \\
.72\end{array}$ \\
\hline $\begin{array}{l}2 \\
2 \\
2 \\
2 \\
2\end{array}$ & $\begin{array}{l}69.20 \\
70.10 \\
71.00 \\
72.50 \\
72.80\end{array}$ & $\begin{array}{l}52.26 \\
53.30 \\
52.43 \\
53.09 \\
52.34\end{array}$ & $\begin{array}{l}53.31 \\
53.33 \\
53.34 \\
53.37 \\
53.37\end{array}$ & $\begin{array}{r}-1.98 \\
-.05 \\
-1.71 \\
-.52 \\
-1.94\end{array}$ \\
\hline $\begin{array}{l}3 \\
3 \\
3 \\
3 \\
3\end{array}$ & $\begin{array}{l}56.95 \\
57.95 \\
60.97 \\
61.48 \\
65.57\end{array}$ & $\begin{array}{l}53.38 \\
53.22 \\
53.18 \\
53.18 \\
53.18\end{array}$ & $\begin{array}{l}53.29 \\
53.28 \\
53.27 \\
53.27 \\
53.28\end{array}$ & $\begin{array}{r}.17 \\
-.12 \\
-.17 \\
-.17 \\
-.19\end{array}$ \\
\hline $\begin{array}{l}3 \\
3 \\
3 \\
3 \\
3\end{array}$ & $\begin{array}{l}65.92 \\
68.77 \\
69.12 \\
70.67 \\
71.38\end{array}$ & $\begin{array}{l}53.18 \\
53.26 \\
53.35 \\
53.43 \\
53.47\end{array}$ & $\begin{array}{l}53.28 \\
53.31 \\
53.31 \\
53.34 \\
53.35\end{array}$ & $\begin{array}{r}-.19 \\
-.09 \\
.07 \\
.18 \\
.23\end{array}$ \\
\hline $\begin{array}{l}3 \\
3 \\
3 \\
3 \\
3\end{array}$ & $\begin{array}{l}73.31 \\
74.95 \\
75.86 \\
77.58 \\
78.68\end{array}$ & $\begin{array}{l}53.60 \\
53.76 \\
53.56 \\
53.72 \\
53.68\end{array}$ & $\begin{array}{l}53.38 \\
53.42 \\
53.45 \\
53.50 \\
53.53\end{array}$ & $\begin{array}{l}.41 \\
.63 \\
.21 \\
.42 \\
.28\end{array}$ \\
\hline $\begin{array}{l}3 \\
3 \\
3 \\
3 \\
3\end{array}$ & $\begin{array}{l}81.13 \\
82.31 \\
82.96 \\
84.79 \\
86.43\end{array}$ & $\begin{array}{l}53.89 \\
53.81 \\
53.89 \\
54.10 \\
54.02\end{array}$ & $\begin{array}{l}53.63 \\
53.68 \\
53.71 \\
53.80 \\
53.89\end{array}$ & $\begin{array}{l}.49 \\
.25 \\
.34 \\
.56 \\
.24\end{array}$ \\
\hline $\begin{array}{l}3 \\
3 \\
3 \\
3 \\
4\end{array}$ & $\begin{array}{l}86.61 \\
86.97 \\
87.32 \\
90.33 \\
70.00\end{array}$ & $\begin{array}{l}54.18 \\
54.06 \\
54.02 \\
54.35 \\
53.40\end{array}$ & $\begin{array}{l}53.90 \\
53.92 \\
53.95 \\
54.15 \\
53.33\end{array}$ & $\begin{array}{l}.51 \\
.25 \\
.14 \\
.36 \\
.14\end{array}$ \\
\hline $\begin{array}{l}4 \\
4 \\
4 \\
4 \\
4\end{array}$ & $\begin{array}{l}74.00 \\
78.00 \\
82.00 \\
86.00 \\
90.00\end{array}$ & $\begin{array}{l}53.57 \\
53.73 \\
53.89 \\
54.06 \\
54.22\end{array}$ & $\begin{array}{l}53.40 \\
53.51 \\
53.66 \\
53.87 \\
54.13\end{array}$ & $\begin{array}{l}.32 \\
.41 \\
.42 \\
.36 \\
.17\end{array}$ \\
\hline
\end{tabular}

For 50 points, Sumpct. $=-0.08$, Rmspct. $=0.81$.
To interpolate onto isotherms, we represent the data for each run with a fourth order power series $(x=T / 100)$,

$$
\bar{C}_{v}=A+B \cdot x+C \cdot x^{2}+D \cdot x^{3}+E \cdot x^{4} .
$$

The first row of table 9 identifies the experimental run. Second and third rows give density in $\mathrm{mol} /$ liter and the molal volume in $\mathrm{cm}^{3} / \mathrm{mol}$ respectively. Following rows give values for the coefficients in (5.3), followed by the rms deviation in percent. We then give selected isotherms of $\bar{C}_{v}$ and of $\bar{C}_{v} / T$, interpolated by use of eqs (5.3).

We obtain coefficients of (5.2) by least squares using the form

$$
\bar{C}_{v} / T=A+B \cdot(k \cdot v),
$$

such that $\left(-d^{2} G / d T^{2}\right) \equiv A$, and $\left(d^{2} P / d T^{2}\right) \equiv B$. Table 10 gives results on selected isotherms. The first three

TABLE 9. Interpolation of $\overline{\mathrm{C}}_{\mathrm{v}}$ on isotherms

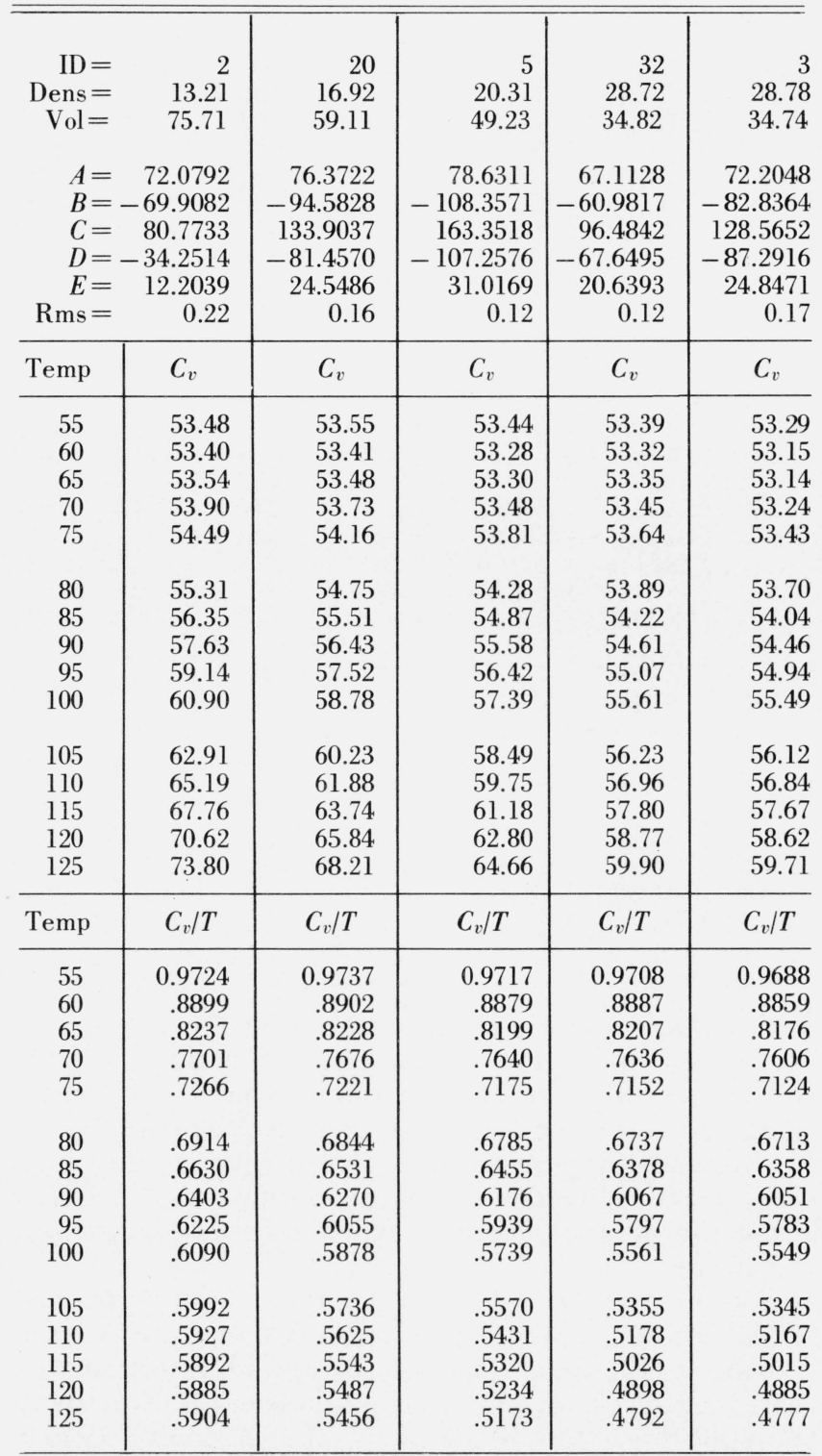


columns give $T, A, B$, for (5.2a). The next column, $D 2 P D T 2$, is the second derivative of Stewart's vapor pressure equation [6]. It is to be compared directly with the values in column B. Columns UA, UB are the absolute, statistical uncertainties in $A, B$, based only on deviations of the data from (5.2a). Maximum uncertainties in the $\bar{C}_{v}$ data of various runs, however, are about 0.5 percent (table 6 ). A probable uncertainty of 0.2 percent may be assigned to each run. One then finds that uncertainties of $B$ for (5.2a) range from about 140 percent at $55 \mathrm{~K}$ to about 2 percent at $125 \mathrm{~K}$. Within these bounds, the vapor pressure equation [6] and our specific heats are consistent at temperatures up to $125 \mathrm{~K}$.

TABle 10. Coefficients A and B for eq (5.2a)

Equation, $C_{v} / T=A+B \cdot(K \cdot V)$

\begin{tabular}{r|r|r|r|r|r}
\hline Temp & $A$ & \multicolumn{1}{|c|}{$B$} & $D^{2} P D T^{2}$ & \multicolumn{1}{c}{$\mathrm{UA}$} & \multicolumn{1}{l}{$\mathrm{UB}$} \\
\hline & & & & & \\
55 & 0.96750 & 0.00077 & 0.00014 & 0.00211 & 0.00039 \\
60 & .88471 & .00074 & .00039 & .00209 & .00039 \\
65 & .81494 & .00116 & .00088 & .00221 & .00041 \\
70 & .75500 & .00198 & .00169 & .00215 & .00040 \\
75 & .70261 & .00314 & .00286 & .00195 & .00036 \\
& & & & & \\
80 & .65613 & .00461 & .00439 & .00170 & .00032 \\
85 & .61438 & .00636 & .00624 & .00145 & .00027 \\
90 & .57646 & .00835 & .00836 & .00122 & .00023 \\
95 & .54175 & .01055 & .01070 & .00105 & .00019 \\
100 & .50978 & .01295 & .01321 & .00093 & .00017 \\
& & & & & \\
105 & .48024 & .01551 & .01586 & .00089 & .00017 \\
110 & .45290 & .01823 & .01862 & .00092 & .00017 \\
115 & .42763 & .02107 & .02148 & .00098 & .00018 \\
120 & .40436 & .02401 & .02445 & .00107 & .00020 \\
125 & .38305 & .02705 & .02753 & .00116 & .00022 \\
\hline
\end{tabular}

\section{Derived Thermodynamic Functions}

We have used (5.1) for integrations along the coexistence path from triple point to critical point, as described in appendix IV. The thermodynamic functions are calculated via the following relations:

$$
\begin{array}{ll}
\Delta S=\int\left[C_{\sigma} / T\right] \cdot d T, & Q=\int C_{\sigma} \cdot d T, \\
\Delta E=Q-\int P \cdot d v, & \Delta H=Q+\int v \cdot d P .
\end{array}
$$

The pressure-volume integrals were evaluated numerically by use of the vapor-pressure equation [6] and the liquid densities of table 1 .

Table 11 presents our results at integral temperatures, at which are calculated the vapor-pressure, the saturated liquid density in $\mathrm{mol} / \mathrm{liter}$, and $C_{\sigma}$ from (5.1) in $\mathrm{J} / \mathrm{mol} \mathrm{K}$. Following columns give the molal increase of entropy, $\Delta S$, the heat absorbed, $C \cdot D T$, the work, $P \cdot D V$, the increase of internal energy, $\Delta E$, the work, $V \cdot D P$, and the increase of enthalpy, $\Delta H$, all relative to the triple-point values. Throughout this table the unit of energy is the joule. Note that
$H-H_{t}=E-E_{t}+P v-(P v)_{t}$, but at the triple point $(P v)_{t}=0.002 \mathrm{~J} / \mathrm{mol}$, hence $\Delta H$ is numerically equal to $\Delta E+P v$.

The probable uncertainty in these functions, obtained by integration, should not exceed that of $C_{\sigma}$ for which we assess a probable error of 0.3 percent, as compared with maximum errors in table 6 from 0.5 to 2.2 percent.

\section{Discussion}

The accurate formulation of adjustment calculations for experimental specific heat measurements on a two-phase system is an exacting challenge $[8,10]$. It therefore may be helpful to record the following details, pointed out by Barieau.

In eq (3.9) the adjustment for vapor entering the capillary tube is not rigorous. This vapor boils away from the liquid phase, and account should be taken of the diminished volume of liquid, an effect of increasing importance at temperatures approaching critical. In place of $\Delta H_{v}$ in eq (3.9) one should use $\Delta H_{a}$, the heat of vaporization per mole of vapor leaving the vessel,

$$
\Delta H_{a}=\Delta H_{v} \cdot \rho_{l} /\left(\rho_{l}-\rho_{g}\right)=T \cdot(d P / d T) / \rho_{g},
$$

where $\rho_{l}, \rho_{g}$ are densities of saturated liquid and vapor. Note that $\Delta H_{a}$ does not vanish at the critical point. An adjustment to present data via eq (7.1), however, is not significant. At $T=152 \mathrm{~K}$, for example, we have roughly $\Delta H_{v}=2000 \mathrm{~J} / \mathrm{mol}, \quad \rho_{l} /\left(\rho_{l}-\rho_{g}\right)=1.7, \quad$ and $\delta N_{c} / \Delta T$ $=2 \cdot 10^{-6} \mathrm{~mol} / \mathrm{deg}$ for our capillary tube.

As noted by H. J. Hoge, several difficult adjustments are eliminated by performing experiments with relatively large and also with relatively small amounts of liquid in the calorimeter [8]. When two phases are present,

$$
\begin{aligned}
C_{\text {total }}= & \operatorname{Lim}(Q / \Delta T)=C_{b}-\left(N-N_{c}\right) \cdot T \cdot \frac{d^{2} G}{d T^{2}} \\
& +V_{b} \cdot T \cdot \frac{d^{2} P}{d T^{2}}+T \cdot \frac{d V_{b}}{d T} \cdot \frac{d P}{d T}+\Delta H_{a} \cdot \frac{d N_{c}}{d T},
\end{aligned}
$$

where $G$ is the Gibbs free energy per mole. The difference of eq (7.2) for two amounts of sample, $N_{2}$ and $N_{1}$, is

$$
C_{2}-C_{1}=-\left(N_{2}-N_{1}\right) \cdot T \cdot d^{2} G / d T^{2} .
$$

From eq (7.3) may be obtained the heat capacity of liquid along the coexistence path by use of $P V T$ data whenever the latter are available,

$C_{\sigma}=T \cdot d S / d T=-T \frac{d^{2} G}{d T^{2}}+\frac{T}{\rho}\left(\frac{d^{2} P}{d T^{2}}\right)-\frac{T}{\rho^{2}} \frac{d \rho}{d T} \cdot \frac{d P}{d T}$,

where $\rho$ and $S$ refer to liquid.

In planning the present work we elected not to follow the above very attractive method. At temperatures approaching the critical temperature, the two 
different filling densities eventually must approach the same value. In this region, $d^{2} G / d T^{2}$ from eq (7.3) might suffer the low accuracy expected in the difference of two large experimental values. Another practical impediment to eq (7.3) is the isothermal condition, which cannot be enforced experimentally for the two different fillings. Interpolation is required along each experimental run. Finally, for specific heats of the liquid at very low temperatures, the above filling densities are not optimum.

TABLE 11. Thermodynamic properties, saturated liquid oxygen

\begin{tabular}{|c|c|c|c|c|c|c|c|c|c|}
\hline$T, \mathbf{K}$ & $P$, atm & $\mathrm{Mol} / 1$ & CSAT & $\Delta S$ & $C \cdot D T$ & $P \cdot D V$ & $\Delta E$ & $V \cdot D P$ & $\Delta H$ \\
\hline 54.351 & 0.001 & 40.842 & 53.313 & 0.000 & 0.00 & 0.000 & 0.00 & 0.000 & 0.00 \\
\hline 55.000 & .002 & 40.752 & 53.306 & .633 & 34.61 & .000 & 34.61 & .001 & 34.61 \\
\hline 60.000 & .007 & 40.057 & 53.274 & 5.270 & 301.05 & .000 & 301.05 & .014 & 301.06 \\
\hline 65.000 & .023 & 39.359 & 53.278 & 9.534 & 567.42 & .001 & 567.41 & .055 & 567.47 \\
\hline 70.000 & .061 & 38.652 & 53.325 & 13.484 & 833.90 & .003 & 833.90 & .155 & 834.06 \\
\hline 75.000 & .143 & 37.936 & 53.423 & 17.166 & 1100.75 & .008 & 1100.74 & .371 & 1101.12 \\
\hline 80.000 & .297 & 37.208 & 53.581 & 20.618 & 1368.23 & .019 & 1368.21 & .786 & 1369.02 \\
\hline 85.000 & .561 & 36.463 & 53.811 & 23.873 & 1636.68 & .042 & 1636.64 & 1.573 & 1638.19 \\
\hline 90.000 & .981 & 35.700 & 54.129 & 26.957 & 1906.49 & .087 & 1906.40 & 2.694 & 1909.18 \\
\hline 90.180 & 1.000 & 35.672 & 54.142 & 27.065 & 1916.23 & .089 & 1916.14 & 2.748 & 1918.98 \\
\hline 95.000 & 1.611 & 34.914 & 54.555 & 29.895 & 2178.15 & .169 & 2177.98 & 4.504 & 2182.65 \\
\hline 100.000 & 2.509 & 34.100 & 55.114 & 32.707 & 2452.26 & .310 & 2451.95 & 7.142 & 2459.40 \\
\hline 105.000 & 3.738 & 33.253 & 55.842 & 35.413 & 2729.57 & .545 & 2729.02 & 10.840 & 2740.41 \\
\hline 110.000 & 5.363 & 32.367 & 56.785 & 38.031 & 3011.03 & .923 & 3010.11 & 15.862 & 3026.90 \\
\hline 115.000 & 7.454 & 31.433 & 58.010 & 40.581 & 3297.89 & 1.517 & 3296.37 & 22.506 & 3320.39 \\
\hline 120.000 & 10.082 & 30.439 & 59.617 & 43.082 & 3591.77 & 2.437 & 3589.33 & 31.118 & 3622.89 \\
\hline 122.000 & 11.300 & 30.022 & 60.398 & 44.074 & 3711.77 & 2.932 & 3708.84 & 35.202 & 3746.97 \\
\hline 124.000 & 12.621 & 29.592 & 61.277 & 45.063 & 3833.42 & 3.518 & 3829.91 & 39.692 & 3873.12 \\
\hline 126.000 & 14.049 & 29.147 & 62.272 & 46.051 & 3956.95 & 4.215 & 3952.74 & 44.621 & 4001.57 \\
\hline 128.000 & 15.591 & 28.686 & 63.402 & 47.041 & 4082.60 & 5.043 & 4077.56 & 50.022 & 4132.62 \\
\hline 130.000 & 17.249 & 28.207 & 64.696 & 48,034 & 4210.67 & 6.029 & 4204.64 & 55.931 & 4266.60 \\
\hline 132.000 & 19.031 & 27.706 & 66.189 & 49.032 & 4341.52 & 7.207 & 4334.31 & 62.390 & 4403.91 \\
\hline 134.000 & 20.942 & 27.181 & 67.930 & 50.040 & 4475.59 & 8.618 & 4466.97 & 69.443 & 4545.04 \\
\hline 136.000 & 22.986 & 26.628 & 69.982 & 51.062 & 4613.45 & 10.318 & 4603.13 & 77.143 & 4690.59 \\
\hline 138.000 & 25.170 & 26.042 & 72.441 & 52.101 & 4755.79 & 12.381 & 4743.41 & 85.548 & 4841.34 \\
\hline 140.000 & 27.501 & 25.416 & 75.443 & 53.164 & 4903.57 & 14.908 & 4888.66 & 94.727 & 4998.30 \\
\hline 142.000 & 29.986 & 24.740 & 79.200 & 54.259 & 5058.06 & 18.041 & 5040.02 & 104.765 & 5162.83 \\
\hline 144.000 & 32.631 & 24.000 & 84.063 & 55.399 & 5221.10 & 21.995 & 5199.11 & 115.766 & 5336.87 \\
\hline 146.000 & 35.448 & 23.177 & 90.653 & 56.602 & 5395.45 & 27.105 & 5368.35 & 127.864 & 5523.32 \\
\hline 148.000 & 38.446 & 22.236 & 100.210 & 57.896 & 5585.66 & 33.950 & 5551.71 & 141.239 & 5726.90 \\
\hline 150.000 & 41.638 & 21.113 & 115.688 & 59.335 & 5800.13 & 43.669 & 5756.46 & 156.158 & 5956.29 \\
\hline 151.000 & 43.312 & 20.445 & 127.975 & 60.142 & 5921.59 & 50.335 & 5871.25 & 164.319 & 6085.91 \\
\hline 152.000 & 45.041 & 19.662 & 146.721 & 61.043 & 6058.19 & 59.059 & 5999.13 & 173.052 & 6231.24 \\
\hline 153.000 & 46.828 & 18.688 & 180.235 & 62.103 & 6219.75 & 71.412 & 6148.34 & 182.485 & 6402.24 \\
\hline 154.000 & 48.675 & 17.292 & 268.103 & 63.503 & 6434.78 & 92.343 & 6342.43 & 192.863 & 6627.64 \\
\hline 154.770 & 50.140 & 13.620 & & 66.148 & 6843.48 & 170.977 & 6672.50 & 202.000 & 7045.48 \\
\hline
\end{tabular}

\section{Appendix I. Volumes of the Calorimeter}

For a spherical shell,

$$
V / V_{1,0}=\left(l / l_{1}\right)^{3} \cdot[1+g(T) \cdot P]^{3},
$$

where $V_{1,0}$ is our reference volume at $T=100 \mathrm{~K}$, $P=0 \mathrm{~atm}$. Length, $l$, may be a diameter $\left(l_{0}\right.$ at $T=0$, $l_{1}$ at $\left.T=100 \mathrm{~K}\right)$ at $P=0$. Function $g(T)$ includes the modulus of elasticity. We developed an approximation formula for thermal expansivity of type 316 stainless steel in the form

$$
l / l_{0}=1+f(T) .
$$

Dropping higher order terms from (1) we then have

$$
V / V_{R}=1+3 \cdot f(T)+3 \cdot g(T) \cdot P,
$$

where

$$
V_{R}=V_{1,0} /[1+3 \cdot f(100)] .
$$

For the elasticity, we use six unpublished values of $(\Delta V \mid \Delta P) / V$ obtained by Younglove and Diller for this 
calorimeter from 40 to $300 \mathrm{~K}$ [3]. They are represented within their precision of a few percent by

$$
(\Delta V / \Delta P) / V=k \cdot x^{1 / 3}, \mathrm{~atm}^{-1},
$$

where $x \equiv T / 100$. Our description for the calorimeter volumes therefore is

$$
V / V_{R}=1+3 \cdot f(T)+k \cdot x^{1 / 3} \cdot P,
$$

where

$$
f(T)=\left[C_{1}+C_{2} \cdot x\right] \cdot \exp [\alpha \cdot(1-1 / x)],
$$

with constants,

$$
\begin{array}{rlrl}
V_{R} & =72.657 \mathrm{~cm}^{3}, & C_{1} & =-0.00021461, \\
C_{2} & =+0.00059455, & \alpha & =1.01062, \\
k & =0.0000111 \mathrm{~atm}^{-1} . &
\end{array}
$$

The reference volume measured by Younglove and Diller was $V_{1,0}=72.74 \mathrm{~cm}^{3}$. We checked this volume at room temperature by expanding oxygen from the bomb at $3 \mathrm{~atm}$ into a calibrated, $130 \mathrm{~cm}^{3}$ glass flask, observing pressures on a commercial, helical-quartz bourdon gage. We used the equation of state derived by L.A.W. for these conditions, $P v / R T=1-0.195 \cdot P / T$, with units of atmospheres and degrees Kelvin. Our volume of $73.19 \pm 0.1 \mathrm{~cm}^{3}$ at $297.3 \mathrm{~K}$ adjusts to $V_{1,0}=72.6 \pm 0.1 \mathrm{~cm}^{3}$. In our specific heat calculations we have used $V_{1,0}=72.74 \mathrm{~cm}^{3}$, assigning an uncertainty of 0.1 percent.

\section{Appendix II. Estimated Temperatures Along the Capillary Tube}

For a capillary tube which is thermally isolated except at its two ends, we may estimate temperatures by use of the temperature-dependent thermal conductivity, $K$, of stainless steel. We developed the approximation formula,

$$
K / K_{100}=\exp \left[1.2 \cdot\left(1-10 / T^{1 / 2}\right)\right] .
$$

We placed (1) in the heat-flow equation, integrated numerically by computer, and developed an approximation formula for the results,

$$
\begin{aligned}
T_{x} & =T_{0} \cdot[1+a \cdot x]^{2 / 3}, \\
a & \equiv\left[\left(T_{1} / T_{0}\right)^{3 / 2}-1\right] .
\end{aligned}
$$

Here $x \equiv l / l_{1}$ is fraction of the distance from the cold end of a capillary tube of length $l_{1}$. Temperature at the cold end is $T_{0}$, at the hot end is $T_{1}$, and at position $x$ it is $T_{x}$.

\section{Appendix III. Method for Estimating Uncertainties (Errors)}

We express the saturated liquid heat capacity in the following form,

$$
C_{\sigma}=(A-B-C-D) / N_{b}+[E-(F-1) \cdot G] \cdot H,
$$

where

$A \equiv C_{a}, \quad B \equiv C_{b}, \quad C \equiv k \cdot T \cdot(d P / d T) \cdot\left(d V_{b} / d T\right)$,

$D \equiv \delta N_{c} \cdot \Delta H_{v} / \Delta T, \quad E \equiv(-d \rho / d T) \cdot(d P / d T) / \rho$,

$F \equiv V_{b} \cdot \rho / N_{b}, \quad G \equiv d^{2} P / d T^{2}, \quad H \equiv k \cdot T / \rho$,

$k=0.101325 \mathrm{~J} / \mathrm{mol}-\mathrm{deg}, \quad \rho$ refers to the liquid phase.

Wherever a sum of terms occurs in brackets we sum absolute errors. For each product or ratio we sum relative errors. Uncertainties in $A, B$, etc., are symbolized $U A, U B$, etc., given in percent at the bottom of table 6 . For $A$, alone, we introduce dependence on the interval, $\Delta T, U_{a}=0.05+0.10 \cdot(5 / \Delta T)$. Results are given in the last two columns of table 6 .

\section{Appendix IV. Definite Integrals Used for Thermal Functions}

Given $A, B, C, r$, and $n$ for eq (5.1), define-

$$
\begin{aligned}
T_{d} & \equiv\left(T_{c}-T_{t}\right), & q & \equiv r^{1 / 2} . \\
x & \equiv\left(T_{c}-T\right) / T_{d}, & z & \equiv x^{1 / 2} .
\end{aligned}
$$

The heat absorbed upon heating from the triple point is -

$\int_{T_{t}}^{T} C_{\sigma} \cdot d T=T_{d} \cdot\left\{2 A \cdot(1-z)+2 B \cdot\left(1-z^{3}\right) / 3+C \cdot \sigma_{D}\right\}$,

where

$$
\sigma_{D} \equiv \sum_{i=1}^{n+1} 2 D_{i} \cdot\left(1-z^{j}\right) / j, \quad j \equiv 2 i-1,
$$

and the binomial coefficients are-

$$
D_{1} \equiv 1, \quad D_{i+1} \equiv-D_{i} \cdot r \cdot(n+1-i) / i .
$$

The increase of entropy upon heating from the triple point is -

$$
\int_{T_{t}}^{T}\left(C_{\sigma} / T\right) \cdot d T=r \cdot\left[A \cdot F_{a}+B \cdot F_{b}+C \cdot \sigma_{E}\right],
$$

where

$$
F_{a} \equiv \lambda / q, \quad F_{b} \equiv[\lambda-2 q \cdot(1-z)] / q^{3},
$$




$$
\begin{aligned}
\lambda & \equiv \ln \left[\frac{1+q}{1-q} \cdot \frac{1-q \cdot z}{1+q \cdot z}\right], \\
\sigma_{E} & \equiv \sum_{i=1}^{n} 2 E_{i} \cdot\left(1-z^{j}\right) / j, \quad j \equiv 2 i-1,
\end{aligned}
$$

and the binomial coefficients are-

$$
E_{1} \equiv 1, \quad E_{i+1} \equiv-E_{i} \cdot r \cdot(n-i) / i .
$$

\section{Appendix V}

The equation of state from reference [6] is

$$
\begin{aligned}
P= & \rho R T+\left(n_{1} T+n_{2}+n_{3} / T^{2}+n_{4} / T^{4}+n_{5} / T^{6}\right) \rho^{2} \\
& +\left(n_{6} T^{2}+n_{7} T+n_{8}+n_{9} / T+n_{10} / T^{2}\right) \rho^{3} \\
& +\left(n_{11} T+n_{12}\right) \rho^{4}+\left(n_{13}+n_{14} / T\right) \rho^{5} \\
& +\rho^{3}\left(n_{15} / T^{2}+n_{16} / T^{3}+n_{17} / T^{4}\right) \exp \left(n_{25} \rho^{2}\right) \\
& +\rho^{5}\left(n_{18} / T^{2}+n_{19} / T^{3}+n_{20} / T^{4}\right) \exp \left(n_{25} \rho^{2}\right) \\
& +\rho^{7}\left(n_{21} / T^{2}+n_{22} / T^{3}+n_{23} / T^{4}\right) \exp \left(n_{25} \rho^{2}\right) \\
& +n_{24} \rho^{n_{28}+1}\left(\rho^{n_{28}}-\rho_{c}^{n_{28}}\right) \exp \left[n_{26}\left(\rho^{n_{28}}-\rho_{c}^{n_{28}}\right)^{2}\right. \\
& \left.+n_{27}\left(T-T_{c}\right)^{2}\right] .
\end{aligned}
$$

The coefficients of the equation of state, $n_{1}$ through $n_{28}$, taken from [6], are given in table 12. These coefficients were determined by a weighted least squares fit of the input experimental data. In this least squares fit, constraints for the critical point were imposed upon the equation of state. These constraints are listed in table 13; the fixed points are listed in table 14.

TABLE 12. Coefficients for the equation

\begin{tabular}{l|l|l}
\hline \hline \multicolumn{3}{c}{$T$ in K, $P$ in atm, $\rho$ in mol $/$ liter } \\
\hline$R=0.0820535$ & $n_{10}=-3.59419602 \times 10$ & $n_{19}=-2.67817667 \times 10^{2}$ \\
$n_{1}=3.38759078 \times 10^{-3}$ & $n_{11}=1.02209557 \times 10^{-6}$ & $n_{20}=1.05670904 \times 10^{5}$ \\
$n_{2}=-1.31606223$ & $n_{12}=1.90454505 \times 10^{-4}$ & $n_{21}=5.63771075 \times 10^{-3}$ \\
$n_{3}=-7.38828523 \times 10^{3}$ & $n_{13}=1.21708394 \times 10^{-5}$ & $n_{22}=-1.12012813$ \\
$n_{4}=1.92049067 \times 10^{7}$ & $n_{14}=2.44255945 \times 10^{-3}$ & $n_{23}=1.46829491 \times 10^{2}$ \\
$n_{5}=-2.90260005 \times 10^{10}$ & $n_{15}=1.73655508 \times 10^{2}$ & $n_{24}=9.98868924 \times 10^{-4}$ \\
$n_{6}=-5.70101162 \times 10^{-8}$ & $n_{16}=3.01752841 \times 10^{5}$ & $n_{25}=-0.00560$ \\
$n_{7}=7.96822375 \times 10^{-5}$ & $n_{17}=-3.49528517 \times 10^{7}$ & $n_{26}=-0.157$ \\
$n_{8}=6.07022502 \times 10^{-3}$ & $n_{18}=8.86724004 \times 10^{-1}$ & $n_{27}=-0.350$ \\
$n_{9}=-2.71019658$ & & $n_{28}=0.90$ \\
\hline
\end{tabular}

TABLE 13. Constraints imposed on the equation of state [6]

$P-\rho-T$ at the critical point $\quad\left\{\begin{array}{l}P=50.14 \mathrm{~atm} \\ \rho=13.333 \mathrm{~mol} / \text { liter } \\ T=154.77 \mathrm{~K}\end{array}\right.$

$(\partial P / \partial \rho)_{T}=0$ at the critical point

$\left(\partial^{2} P / \partial \rho^{2}\right)_{T}=0$ at the critical point

$(\partial P / \partial T)_{\rho}=d P / d T$ from appendix 6 at the critical point $=1.928386 \mathrm{~atm} / \mathrm{K}$
TABLE 14. Fixed point data [6]
Critical pressure

Critical temperature

Critical density

Density saturated vapor at nbp

Density saturated liquid at nbp

Triple point pressure

Triple point temperature
Normal boiling temperature (IPTS, fixed point)
50.14 atm

$154.77 \mathrm{~K}$

$13.333 \mathrm{~mol} / \mathrm{liter}$

$90.18 \mathrm{~K}$

$0.1396 \mathrm{~mol} / \mathrm{liter}$

$35.65 \mathrm{~mol} / \mathrm{liter}$

$0.00150 \mathrm{~atm}$

$54.353 \mathrm{~K}$

\section{Appendix VI. The Vapor Pressure Equation from [6]}

The vapor pressure equation is $[P$ in atm, $T$ in $K]$ :

$\ln P=a_{0}+a_{1} T+a_{2} T^{2}+a_{3} T^{3}+a_{4} T^{4}+a_{5} T^{5}+a_{6} T^{6}+a_{7} T^{7}$.

TABLE 15. Coefficients for the vapor pressure equation

\begin{tabular}{l|l}
\hline \hline & \\
$a_{0}=-6.25967185 \times 10$ & $a_{4}=-4.09349868 \times 10^{-6}$ \\
$a_{1}=2.47450429$ & $a_{5}=1.91471914 \times 10^{-8}$ \\
$a_{2}=-4.68973315 \times 10^{-2}$ & $a_{5}=-5.13113688 \times 10^{-11}$ \\
$a_{3}=5.48202337 \times 10^{-4}$ & $a_{7}=6.02656934 \times 10^{-14}$ \\
\hline
\end{tabular}

\section{References}

[1] Woolley, H. W., Thermodynamic properties of molecular oxygen, ASTIA Document AD 19900 (June 1953).

[2] Goodwin, R. D., Apparatus for determination of $P V T$ relations and specific heats of hydrogen to $350 \mathrm{~atm}$ at temperatures above $14{ }^{\circ} \mathrm{K}$, J. Res. NBS 65C (Engr. and Instr.) No. 4, 231-243 (1961).

[3] Younglove, B. A., and Diller, D. E., The specific heat of saturated liquid parahydrogen from 15 to $32^{\circ} \mathrm{K}$, Cryogenics 2 (No. 5), 283 (1962).

[4] Giauque, W. F., and Johnston, H. L., The heat capacity of oxygen from $12{ }^{\circ} \mathrm{K}$ to its boiling point and its heat of vaporization. The entropy from spectroscopic data. J. Am. Chem. Soc. 51, 2300-2321 (1929).

[5] Scott, R. B., Meyers, C. H., Rands, R. D., Jr., Brickwedde, F. G., and Bekkedahl, N., Thermodynamic properties of 1,3-butadiene in the solid, liquid, and vapor states, J. Res. NBS 35, 39-85 (1945) RP1661.

[6] Stewart, R. B., The Thermodynamic Properties of Oxygen, Dissertation, Dept. of Mech. Engr'g., Univ. of Iowa, June 1966.

[7] Muijlwijk, R., Moussa, M. R., and Van Dijk, H., The vapour pressure of liquid oxygen, Physica 32, 805 (1966).

[8] Hoge, H. J., Heat capacity of a two-phase system, with applications to vapor corrections in calorimetry, J. Res. NBS 36, 111 (1946) RP1693.

[9] Walker, P. A., The equation of state and specific heat of liquid argon, Thesis, Queen Mary College, Univ. of London (1956).

[10] Rowlinson, J. S., Liquids and Liquid Mixtures (Butterworths, London. 1959).

[11] Eucken, A., Uber das thermische Verhalten einiger komprimierter und kondensierter Gase bei tiefen Temperaturen, Verhandl. Ḍeut. Physik. Ges. 18, 4-17 (1916).

[12] Clusius, K., Uber die spezifische Wärme einiger kondensierter Gase zwischen $10^{\circ}$ abs und ihrem Tripelpunkt, Z. physik. Chem. (Leipzig) B3, 41-79 (1929).

[13] Van Itterbeek, A., and Van Dael, W., Properties of some cryogenic liquids from sound velocity data, Advances in Cryogenic Engineering 9, 207-216 (1963).

[14] Barieau, R. E., Helium Research Center, U.S. Bureau of Mines, Amarillo, Texas 79106, private communication, Jan. 14, 1965.

[15] Yang, C. N., and Yang, C. P., Critical point in liquid-gas transitions, Phys. Rev. Letters 13(9), 303-305 (1964). 
[16] Barieau, R. E., Thermodynamic properties of a van der Waals fluid in the two-phase region, U.S. Bureau of Mines Report RI 6950, June 1967.

[17] Hoge, H. J., Vapor pressure and fixed points of oxygen and heat capacity in the critical region, J. Res. NBS 44, 32l-345 (1950) P.P2081.
[18] Weber, L. A., Thermodynamic and related properties of oxygen from the triple point to $300 \mathrm{~K}$ at pressures to 330 atmospheres, internal report, June 20, 1968.

(Paper 73Al-529) 